\title{
Search for Cosmic Particles with the Moon and LOFAR
}

T. Winchen ${ }^{1, \star}$, A. Bonardi ${ }^{2}$, S. Buitink ${ }^{1}$, A. Corstanje $^{2}$, J. E. Enriquez ${ }^{2}, H$. Falcke F, $^{2,7}$, J. R. Hörandel $^{2}$, P. Mitra ${ }^{1}$, K. Mulrey ${ }^{1}$, A. Nelles ${ }^{3}$, J. P. Rachen ${ }^{2}$, L. Rossetto ${ }^{2}$, P. Schellart ${ }^{2}, O$. Scholten $^{4 \partial}$, S. Thoudam ${ }^{2}$, T.N.G. Trinh ${ }^{4,6}$, and S. ter Veen ${ }^{5}$ (The LOFAR Cosmic Ray KSP)

${ }^{1}$ Vrije Universiteit Brussel (Belgium)

${ }^{2}$ Radboud University Nijmegen (The Netherlands)

${ }^{3}$ University of California Irvine (USA)

${ }^{4} \mathrm{KVI}-\mathrm{CART}$ (The Netherlands)

${ }^{5}$ ASTRON (The Netherlands)

${ }^{6}$ University of Groningen (The Netherlands)

${ }^{7}$ NIKHEF (The Netherlands)

\begin{abstract}
The low flux of the ultra-high energy cosmic rays (UHECR) at the highest energies provides a challenge to answer the long standing question about their origin and nature. A significant increase in the number of detected UHECR is expected to be achieved by employing Earth's moon as detector, and search for short radio pulses that are emitted when a particle interacts in the lunar rock. Observation of these short pulses with current and future radio telescopes also allows to search for the even lower fluxes of neutrinos with energies above $10^{22} \mathrm{eV}$, that are predicted in certain Grand-Unifying-Theories (GUTs), and e.g. models for super-heavy dark matter (SHDM). In this contribution we present the initial design for such a search with the LOFAR radio telescope.
\end{abstract}

\section{Introduction}

The extremely low flux of ultra-high energy cosmic rays (UHECR) makes the search for their sources a challenging task (e.g. [1]). The same challenge arises in testing specific theories of grandunification [2] and super heavy dark matter [3, 4] by their predicted flux of neutrinos with extreme energies beyond $1 \mathrm{ZeV}$. To test these theories and increase the available UHECR data at the highest energies, detectors several orders of magnitude larger than currently available are required. One approach to achieve this necessary increase of detector area is to use Earth's Moon as detector. The Moon is 'read-out' by existing radio telescopes that record the nanosecond radio pulses that are created when a particle interacts in the lunar rock.

Several searches with radio telescopes operating in the $\mathrm{GHz}$ frequency regime have so far yielded only upper limits on the neutrino flux and have not been sensitive enough to observe the low UHECR flux [5- [9]. The sensitivity of these searches was in particular limited by the frequency range of the used telescopes that operate in the $\mathrm{GHz}$ regime. While at $\mathrm{GHz}$ frequencies the expected pulse amplitudes reach a maximum, the pulses cannot escape the moon for most part of its surface; only

^e-mail: tobias.winchen@rwth-aachen.de 


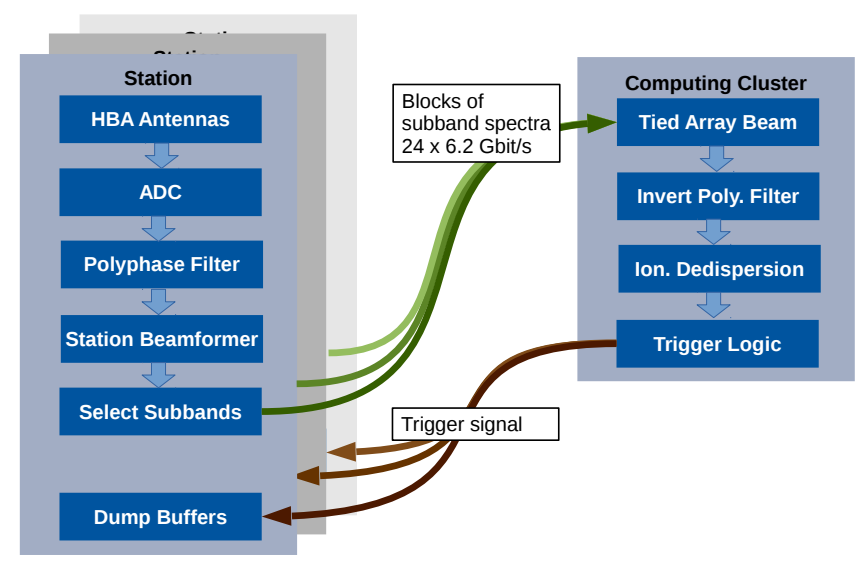

Figure 1. Overview of the online data analysis processing steps for the detection of ns-pulses with LOFAR.

pulses from particles hitting the limb are detectable at Earth. Conversely, similar searches with the LOw Frequency ARray (LOFAR) [10] can use the full visible lunar surface as sensitive area, as it operates in a frequency range that allows pulses to escape the lunar rock in more geometries and is optimal for the lunar detection of particles [11].

\section{Signal Processing at LOFAR}

LOFAR consists of several thousand omni-directional antennas located in more than 48 stations distributed throughout European. Twenty-four of the stations are grouped into a dense core of approximately $2 \mathrm{~km}$ diameter in the Netherlands. All stations are equipped with 96 low-band antennas (LBAs) with a frequency range from 10-90 MHz and at least 48 'tiles' of 16 high-band antennas (HBAs) each, with a frequency range from 110-240 MHz. By beamforming, i.e. coherent stacking the signals of the individual antennas with a corresponding delay, the sensitivity of a station is tuned towards a specific direction. A station thus assumes the role of a single dish in a classical radio telescope.

As preparation for astronomical observations, the data is preprocessed in several steps in LOFAR before it can be accessed by the user. An overview of the default processing steps together with the online-analysis steps required here is given in figure 1. After digitization, the data is first processed by a polyphase filter (PPF), which splits the data stream into 256 sub-channels of $195 \mathrm{kHz}$ width. Compared to splitting data with a Fourier transformation, the PPF allows efficient sampling conversion while preventing signal-leakage between neighboring channels, thus allowing precise filtering. However, within the individual sub-channels the time resolution is reduced from $5 \mathrm{~ns}$ to only a few micro-seconds due to critical down-sampling. The filtered data of the individual antennas of one station is then combined into a 'station beam' before it is transmitted to a computing cluster for further processing.

While the pre-filtering of the signal is convenient for typical astronomical observations, here we require the full nano-second resolution. The thus necessary inversion of the PPF is a lossy process, but we can use the thereby approximately reconstructed signal to trigger storage of the raw voltage traces which are buffered for 5 seconds. We therefore will first combine the station beams to several tight-array beams pointing to different spots on the moon, and then construct nanosecond time traces 
from the combined signals. These are then corrected for ionospheric dispersion, and eventually trigger a read-out of the buffer board.

\section{Polyphase Synthesis}

The polyphase filter analysis is an efficient implementation of an multi channel Finite-ImpulseResponse (FIR) filter with corresponding optimization of the sampling rate to split the signal $x[n]$ into well defined sub-bands $\hat{y}_{i}[m]$, with $i=0 \cdots M$. The polyphase-filter mathematically corresponds to the Fourier transform $\mathcal{F}(\mathbf{y})$ of the transformed signal $\mathbf{y}=H \cdot \mathbf{x}$, with matrix $H$ corresponding to the single-channel filter. For a K-tap filter into M-sub-channels $H$ is a $K \cdot M \times M$ matrix. The filter principle is sketched in figure 2

Inversion of the Fourier transformation in the polyphase analysis is possible without loss of information. The inverse of the filter matrix $H^{-1}$, however, does not exists as $H$ is not square. While methods exists (e.g. [12]) to construct an approximate inverse $G$ so that $G \cdot H=1$, the one-step inversion produces echos of short pulses as e.g. in [13]. This limits their usability here, as we thus expect a large number of false triggers generated from RFI by this method. We here therefore investigate a more robust but computationally more complex iterative method to solve the linear-systems of equations $H \cdot \mathbf{x}=\mathbf{y}$.

As the system is under-determined, a side condition is required to constrain the solutions. The reason for the ill-determination of the inverse is here, that any $x[n]$ influences $y_{i}[m]$ for $K$ values of $m$. Thus, for a filtered signal of finite length, $K \times M$ samples at the beginning, respectively end, of the reconstructed input signal are not completely determined. Searching for the minimum solution $\|\min x\|$ can thus be used as side condition here, as this minimizes the signal in the under-determined areas at cost of a slight damping of the reconstructed signal.

An algorithm using this side condition to solve under-determined linear systems is the LSMR algorithm [14]. Compared to alternatives as e.g. the LSQR algorithm, the LSMR algorithm has also the advantage of practically monotonically conversion that allows for safe early termination, which might be advantageous for a real-time application.

In figure 3 an example of the reconstruction of a trace with a prototype implementation of this method is given. The input signal shown in fig. 3 (a) is composed of white noise and delta peaks of fixed height at random time sets. After applying a polyphase filter and its inverse to the trace, the signal is recovered almost perfectly, except for the edges of the trace as shown in fig. 3 (b). The differences between the input signal and recovered signal is exactly zero except for small time windows at intervals corresponding to the number of sub-channels as shown in fig. 3 ( c, d). As these

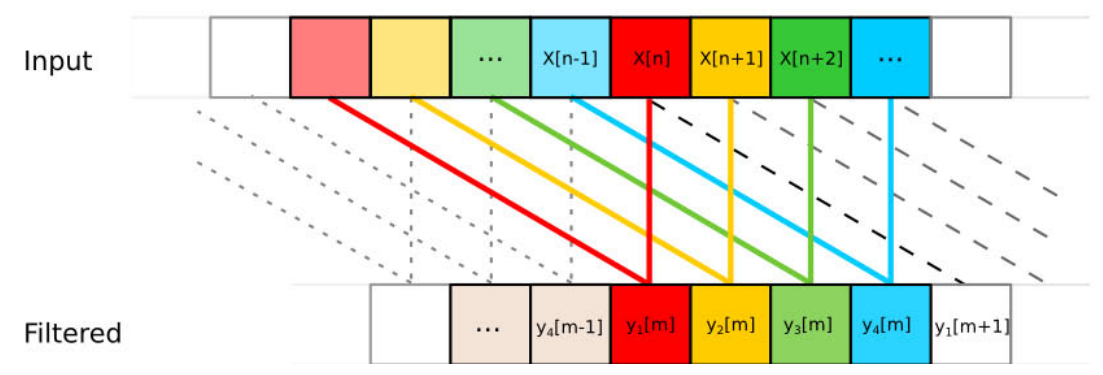

Figure 2. Sketch of the filter stage of an 2-tap 4-channel PPF before application of a 4-point FFT to $\mathbf{y}$. 

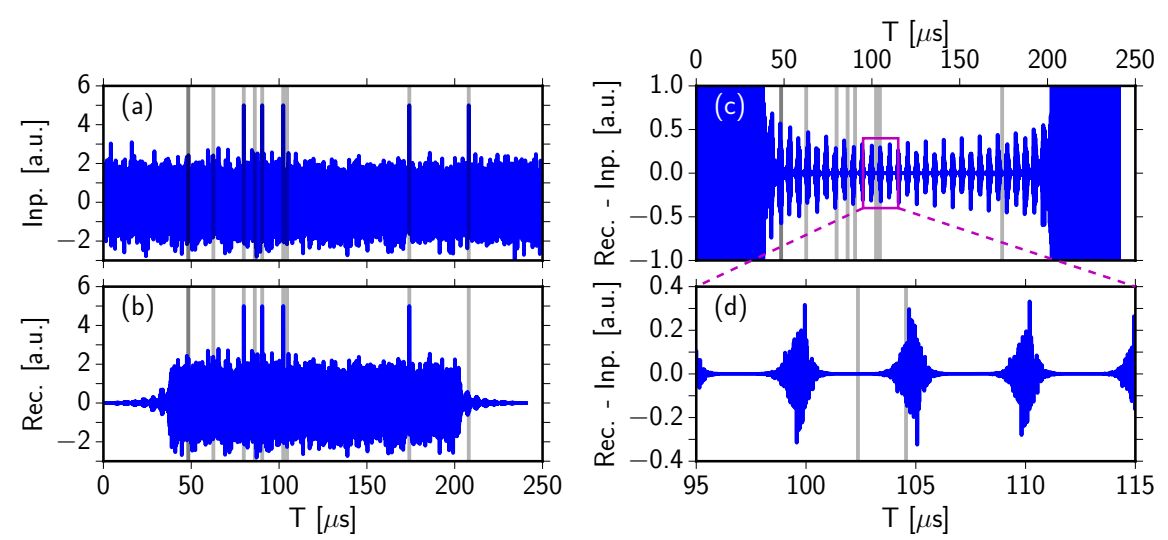

Figure 3. Accuracy of PPF-Synthesis. (a) Input Signal, (b) reconstructed signal, (c) difference between input and reconstructed signal, (d) zoom into (c). The position of input pulses are marked by vertical gray lines.

windows are known, a necessary increase of the trigger sensitivity is limited to a small time intervall. In particular, the uncertainty in the reconstruction is not related to the position of the input pulses and thus not critical for the trigger. The maximum amplitude found here corresponds to less than $30 \%$ of the root-mean squared of the white noise.

\section{Conclusion}

The LOFAR radio telescope is a promising instrument for the search of cosmic-particle induced radiopulses from Earth's moon. Such a search requires triggering on preprocessed data and in particular the reconstruction of nano second resolution time traces via a polyphase synthesis. The iterative approach to polyphase synthesis investigated here is suitable for triggering the data, as it does not introduce artefacts that are likely to increase the trigger rate.

\section{References}

[1] K. Kotera, A.V. Olinto, Annual Review of Astronomy and Astrophysics 49, 119 (2011)

[2] P. Bhattacharjee, AIP Conf. Proc. 433, 168 (1998)

[3] R. Aloisio, V. Berezinsky, M. Kachelriess, Phys. Rev. D74, 023516 (2006)

[4] R. Aloisio, S. Matarrese, A.V. Olinto, JCAP 1508, 024 (2015)

[5] S. Buitink et al., Astron. Astrophys. 521, A47 (2010)

[6] T.H. Hankins, R.D. Ekers, J.D. O’Sullivan, MNRAS 283, 1027 (1996)

[7] C.W. James et al., MNRAS 410, 885 (2011)

[8] C.W. James et al., MNRAS 379, 1037 (2007)

[9] J.D. Bray et al., Astropart. Phys. 65, 22 (2014)

[10] M.P. van Haarlem et al., Astronomy and Astrophysics 556, A2 (2013)

[11] O. Scholten et al., Astroparticle Physics 26, 219 (2006)

[12] M. Unser, Proceedings of the IEEE 88, 569 (2000)

[13] K. Singh et al., Nucl. Instrum. Meth. A664, 171 (2012)

[14] D. Fong, M. Saunders, SIAM Journal on Scientific Computing 33, 2950 (2011) 